\title{
PROBLEMÁTICA SOCIAL Y EFECTOS DE LA SUSTITUCIÓN DE LOS CULTIVOS DE COCA POR CACAO EN EL MUNICIPIO DE PAUNA (BOYACÁ)
}

Lina Marleny López Sánchez Universidad Pedagógica y Tecnológica de Colombia Colombia

Erika Alejandra Fúquene Universidad Pedagógica y Tecnológica de Colombia Colombia

Karen Lorena Montenegro Universidad Pedagógica y Tecnológica de Colombia Colombia

Gissel Lorena Ramirez Universidad Pedagógica y Tecnológica de Colombia Colombia

Angélica María Sánchez Pineda Universidad Pedagógica y Tecnológica de Colombia Colombia 
Panorama Económico, Vol. 26 - No. 4 (Octubre - Diciembre de 2018), pp. 537- 560

\author{
Lina Marleny López Sánchez \\ Gissel Lorena Ramirez \\ Erika Alejandra Fúquene \\ Angélica María Sánchez Pineda
}

Karen Lorena Montenegro

Problemática social y efectos de la sustitución de los cultivos de coca por cacao en el municipio
de Pauna (Boyacá)

\title{
Resumen
}

El municipio de Pauna, en el occidente de Boyacá, se ha visto afectado por la violencia, la pobreza, el rechazo social y el abandono institucional, entre otros flagelos, debido a la siembra de cultivos ilícitos (coca) y a la presencia de grupos armados al margen de la ley; sin embargo, alrededor de 1200 familias campesinas de esta región luchan por cambiar esta lúgubre historia. Por eso, esta investigación se refiere a la problemática social y a los efectos de la sustitución de los cultivos de coca por cacao que se adelanta en esa población desde el 2004 en el marco del Plan Colombia. Se identificaron los factores que originaron la sustitución de los cultivos ilícitos, así como sus consecuencias socioeconómicas, mediante la aplicación de instrumentos tales como entrevistas, encuestas y observación directa. El estudio se apoya en un marco de referencia teórico-conceptual, centrado en la economía campesina y la agricultura familiar, y en el marco legal de la erradicación de cultivos ilícitos, entre otros referentes. Esta investigación exploratoria emplea la metodología de estudio caso, a través de un análisis descriptivo de estos hechos y sus respectivos componentes. Se concluye mostrando el aporte del Plan Colombia a las comunidades campesinas en estudio, y resaltando la necesidad de contar con el apoyo gubernamental a las iniciativas de los cacaoteros de Pauna y sus organizaciones asociativas para consolidar estos procesos de reconversión productiva.

Palabras clave: Cultivo ilícito, problemática social, cultivo de cacao, economía campesina, agricultura campesina.

\section{Problèmes sociaux et effets de la substitution des cultures de cacao au cacao dans la commune de Pauna (Boyacá)}

\section{Résumé}

La municipalité de Pauna, dans l'ouest de Boyacá, a été touchée par la violence, la pauvreté, le rejet social et l'abandon institutionnel, entre autres fléaux, en raison de la plantation de cultures illicites (coca) et de la présence de groupes armés illégaux; Cependant, environ 1200 familles paysannes de cette région luttent pour changer cette lugubre histoire. C'est pourquoi cette recherche porte sur la problématique sociale et sur les effets de la substitution des cultures de coca par du cacao qui se développe dans cette population depuis 2004 dans le cadre du Plan Colombie. Les facteurs à l'origine de la substitution des cultures illicites et leurs conséquences socioéconomiques ont été identifiés à l'aide d'outils tels que des entretiens, des enquêtes et des observations directes. L'étude s'appuie sur un cadre de référence théorique et conceptuel, centré sur l'économie paysanne et l'agriculture familiale, et sur le cadre juridique de l'éradication des cultures illicites, entre autres. Cette recherche exploratoire utilise la méthodologie d'étude de cas, à travers une analyse descriptive de ces faits et de leurs composantes respectives. On conclut en montrant la contribution du Plan Colombie aux communautés paysannes à l'étude, et soulignant la nécessité d'un soutien gouvernemental aux initiatives des producteurs de cacao de Pauna et de leurs organisations associatives pour consolider ces processus de reconversion productive.

Mots clés: Culture illicite, problématique sociale, culture du cacao, économie paysanne, agriculture paysanne.

\section{Social problems and effects of the substitution of coca crops for cocoa in the municipality of Pauna (Boyacá)}

\begin{abstract}
The municipality of Pauna, west of Boyacá state, has been involved in violence, poverty, social rejection and institutional abandonment, among others, due to the sowing of illicit crops (coca) and the presence of armed groups outside the law; however, around 1,200 peasant families in this region struggle to change this grim history. For that reason, this research refers to social problems and the effects of the substitution of coca crops for cocoa, which have been moving ahead since 2004, derived from Plan Colombia. Factors that gave rise to the substitution of illicit crops were identified, as well as their socio-economic consequences, through application of instruments, such as interviews, surveys and direct observation, supported in a theoretical-conceptual framework, centered on the peasant economy and family agriculture, and in a legal framework on eradication of illicit crops, among other references. This exploratory research uses case-study methodology, which is carried out through a descriptive analysis of these facts and their components. The study concludes by showing the contribution of Plan Colombia to the peasant communities of Pauna, and highlighting the need of governmental support to these productive reconversion processes.
\end{abstract}

Keywords: Illicit crops, social problems, cocoa crops, peasant economy, peasant agriculture. 


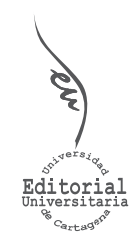

Artículo de Investigación

\section{Problemática social y efectos de la sustitución de los cultivos de coca por cacao en el municipio de Pauna (Boyacá)}

INFORMACIÓN DEL ARTÍCULO

Recepción del artículo: 02/01/2018

Concepto de evaluación: 07/03/2018

Aceptación del artículo: 17/06/2018
Lina Marleny López Sánchez* Universidad Pedagógica y Tecnológica de Colombia

Erika Alejandra Fúquene** Universidad Pedagógica y Tecnológica de Colombia, Colombia

Karen Lorena Montenegro** Universidad Pedagógica y Tecnológica de Colombia, Colombia

Gissel Lorena Ramirez** Universidad Pedagógica y Tecnológica de Colombia, Colombia

Angélica María Sánchez Pineda*** Universidad Pedagógica y Tecnológica de Colombia, Colombia

\section{INTRODUCCIÓN}

Desde hace más de medio siglo Colombia ha sido azotada por un conflicto armado interno, en gran parte derivado del narcotráfico y de los grupos al margen de la ley, lo que ha puesto a la población rural en situación de vulnerabilidad, principalmente en aquellas zonas donde se han extendido los cultivos ilícitos. Frente a ello, se han planteado diferentes estrategias por parte del Gobierno nacional, con el fin de mitigar esta problemática en muchos departamentos que han sido históricamente el escenario de estos conflictos sociales.

\footnotetext{
*Contadora pública, magíster en Administración, especialista en Revisoría Fiscal, profesora e investigadora asociada de la Escuela de Contaduría Pública de la Universidad Pedagógica y Tecnológica de Colombia, Tunja, Colombia. Directora del Grupo de Investigación Dinámica Contable (GIDICON).

** Grupo de Investigación Dinámica Contable (GIDICON) de la Universidad Pedagógica y Tecnológica de Colombia ***Programa de Ingeniería Agronómica de la Universidad Pedagógica y Tecnológica de Colombia, integrante del Grupo de Investigación Dinámica Contable (GIDICON).
} 
Boyacá ha sido uno de los departamentos donde ha tenido lugar dicha problemática, tal ha sido el caso de la provincia de Occidente, en municipios como Buenavista, Maripí, Muzo, Otanche, San Pablo de Borbur, Pauna, Quípama, Briceño y La Victoria. Por otra parte, en este territorio, hacia los años 70 y 80 se dio el auge esmeraldero, también llamado "fiebre verde", cuando muchos agricultores dejaron sus fincas en busca de hacer dinero o "enguacarse" en alguna de las cuatro minas de la región; algunos lograron sacar provecho, mientras que otros no contaron con la misma suerte.

Adicionalmente, organizaciones armadas, movidas por el interés de continuar haciendo dinero de manera ilícita, encontraron en el cultivo de coca una alternativa que al parecer era muy rentable. Así tomaron el control de estos territorios, obligando a los pobladores a sembrar coca, como una alternativa tendiente a mejorar su nivel de vida. Estos cultivos marcaron una época de violencia, en que se juntaron explotaciones esmeralderas y narcocultivos, lo que trajo una escalada de violaciones a los derechos humanos, desarraigo y desconfianza. Es ahí donde se inicia un proceso de intervención social en la provincia de Occidente de Boyacá, liderado por la Diócesis de Chiquinquirá, que buscaba reemplazar la cultura del dinero fácil, por la defensa de una vida digna y en paz en sus territorios.

En la provincia se logran constituir y fortalecer diez organizaciones compuestas por agricultores, con deseos de sustituir de manera voluntaria los cultivos de coca por cacao. En Pauna se creó la Asociación de Productores de Cacao (Aprocampa), la cual se enfocó en buscar objetivos comunes, teniendo como eje un mayor desarrollo, que le permitiera posicionar el cultivo del cacao dentro de los mejores mercados nacionales.

Este proceso de sustitución estuvo acompañado tanto de apoyo nacional como internacional, tal como el Plan Colombia y el programa de Familias Guardabosques. Este plan busca la erradicación del cultivo de la coca y su sustitución por el cacao, además de dar protección a las zonas de bosque. Las ayudas ofrecidas por el Estado en insumos agropecuarios, asistencia técnica y herramientas, apuntaban a un único fin: lograr erradicar todos los cultivos de coca en la provincia del Occidente de Boyacá. Así, los municipios de Pauna y San Pablo de Borbur lograron ser reconocidos como los primeros municipios libres de coca del país.

Lo anterior plantea algunos interrogantes: ¿Cuál ha sido el papel del Estado en el programa de sustitución de cultivos de coca? ¿Después de erradicados los cultivos de coca, ha mejorado el bienestar de la población? ¿Ha sido suficiente el apoyo del Gobierno nacional para garantizar la continuidad del proceso de erradicación de los cultivos ilícitos? El propósito central de este documento es analizar la problemática social y los efectos resultantes de la sustitución de los cultivos de coca por cacao, en el contexto del Plan Colombia, que se ha venido adelantando en Pauna (Boyacá).

\section{MARCO TEÓRICO}

\section{La economía campesina}

La problemática de la economía campesina se remonta a comienzos del siglo XX, siendo Alexander Chayanov uno de los estudiosos más destacados. Este economista y agrónomo ruso, perteneciente a la escuela de organización y producción, marcó un hito con su obra La organización de la unidad económica campesina (Chayanov, 1974). 
Este autor parte del análisis de la organización y el funcionamiento de las unidades de producción de la agricultura familiar en el marco del desarrollo agrícola capitalista, particularmente de lo que se denomina la economía campesina, forma de organización que coexiste con otras formas sociales dentro de un modo de producción. Según Chayanov (1974), la economía campesina no utiliza trabajo asalariado, sino familiar, combinando la explotación de la tierra con otras actividades artesanales y comerciales. Su énfasis radica en la producción destinada al autoconsumo.

Diez (2013) afirma que para Chayanov:

uno de los rasgos distintivos, esto es que marcan la especificidad de la 'economía campesina', es el carácter familiar de la unidad de trabajo, como así también, el cálculo económico particular de este tipo de unidades que consiste en el logro de una relación de equilibrio entre el consumo familiar-los bienes necesarios para satisfacerlo- y el esfuerzo que ello requiere. (p. 155)

Por otro lado, Gonzalves (2007, p.13) sostiene que "la economía campesina NO es un modo de producción precapitalista (modo de producción mercantil) que habría antecedido al modo de producción capitalista y que hasta ahora se estaría manteniendo de manera atípica en la economía". Párrafo adelante este autor añade: "(...) la economía campesina es un modo de producción con su lógica interna, autónoma y propia, que se articula externamente con el capitalismo, la economía campesina (economía mercantil) 'no es una totalidad social'". De allí que la economía campesina está expuesta a su desaparición si no se encuentran formas asociativas de producción con un elevado componente familiar.

En contraste, la corriente del pensamiento económico dominante hace apología de la economía empresarial agroindustrial, que plantea la competitividad de los mercados globalizados, que exige la aparición de cadenas de valor producción-distribuciónconsumo agroalimentario (Esteve, 2009). Pese a que la economía campesina utiliza mano de obra familiar, acude a la sabiduría ancestral e innovaciones empíricas, se desenvuelve en condiciones de precariedad y múltiples riesgos, asociados al cambio climático, aportes a la economía local, mercados inestables, capacidad de negociación y restricciones en la información, entre otros problemas. No obstante, la economía campesina sobrevive, coexistiendo con la gran empresa agrícola capitalista moderna (Sevilla \& González, 2004).

Gonzalves, a su vez, citando a Bartra (1982), anota: "entendemos por unidad económica campesina a una célula de producción y consumo, constituida por la unidad orgánica de fuerza de trabajo y medios de producción" (p. 14), subrayando que "es una característica de la economía campesina que ambos procesos estén integrados: consumo y producción son en muchos casos actividades conjuntas (...) es una diferencia sustantiva con la económica capitalista".

Por otro lado, para Figueroa (citado por Gonzalves, 2007, p. 15): "la familia campesina es una unidad de consumo y de producción a la vez"; y agrega que "la economía campesina parece mostrar un comportamiento económico motivado por asegurar una cierta independencia con la autosubsistencia", destinando parte de la producción al mercado y la posibilidad de convertirse en asalariado.

Algunas de las características presentes en la economía campesina son: 
Tabla 1.

Características de la economía campesina

\begin{tabular}{lcc}
\hline \multicolumn{1}{c}{ Características } & $\begin{array}{l}\text { Economía } \\
\text { campesina }\end{array}$ & $\begin{array}{c}\text { Economía } \\
\text { capitalista }\end{array}$ \\
\hline Separación productodirecto / medios de producción & No & Sí \\
$\begin{array}{l}\text { Trabajo asalario (fuerza de trabajo- mercancías) / } \\
\text { explotación, producción destinada al mercado }\end{array}$ & $\begin{array}{c}\text { No } \\
\text { Excedente }\end{array}$ & Sí \\
Factor clave: acumulación de capital & No & Sí \\
Integración producción consumo & Sí & No
\end{tabular}

Fuente: Elaboración propia, a partir de Gonzalves (2007)

\section{Características de la agricultura familiar}

En el presente siglo ha adquirido relevancia el análisis de la denominada agricultura familiar, concepto emparentado con la economía campesina.

Martínez y Acevedo (2016) señalan: "Estudios de organismos internacionales concluyen que cerca del $70 \%$ de los alimentos en el mundo los producen familias agricultoras y el $40 \%$ de los hogares dependen de este tipo de agricultura como forma de vida" (p. 15). De acuerdo con este autor,

La agricultura familiar está en todas las actividades de producción agrícola, pecuaria, forestal, pesquera, pastoril y acuícola. Se caracteriza por el uso preponderante de fuerza de trabajo familiar, acceso limitado a recursos de tierra y capital, la actividad agrícola es la principal fuente de ingresos de la familia. La agricultura familiar está ligada de manera indisoluble a la seguridad y soberanía alimentaria, rescata los alimentos tradicionales, contribuye a la protección de la biodiversidad agrícola y al uso sostenible de los recursos naturales. (p. 15)

El concepto de agricultura familiar es diferente al de economía campesina, "quizá abarcándolo, debido a que resalta las distintas actividades económicas, sociales, culturales, ambientales que cumplen el agricultor y especialmente el grupo familiar ligado a la ruralidad" (Martínez \& Acevedo, 2016p. 17). Más adelante, este autor señala:

en Colombia, por mucho tiempo, han hecho carrera conceptos como 'pequeño productor y economía campesina, sobre los que es necesario hacer algunas reflexiones. La economía campesina 'define diversas actividades económicas que realizan los actores rurales, especialmente los pequeños productores'; por tanto, es un concepto delimitado por el análisis económico. El concepto de agricultura familiar busca llamar la atención sobre el conjunto de actividades realizadas por un actor específico: la familia. Si bien los conceptos de agricultura familiar y economía campesina no son contradictorios y comparten el aporte que hacen las familias del campo, presentan dimensiones y énfasis particulares: la economía campesina resalta el aspecto económico. (p. 17) 
Desde otro ángulo, Diez (2013) revela un aspecto poco conocido, la interrelación de la producción familiar con el mercado, al plantear que

La familia como base de la organización agrícola ya fue abordado en la sociología y antropología rural, lo que aporta es un estudio de la heterogeneidad de este segmento, y el análisis de los grados de integración de estos agricultores familiares al mercado, ya que este impulso de transformación tiene que ver en gran medida con la relación con la agroindustria en el escenario internacional. (2013, p. 158)

En síntesis, los autores mencionados reconocen la persistencia de la economía campesina y la aparición de la agricultura familiar como una modalidad vinculada a la economía capitalista. En este contexto, la economía campesina hace su transición o conversión a la agricultura familiar.

\section{Los alcances del desarrollo rural}

El manejo de los problemas agrarios ha seguido una trayectoria muy cambiante, acorde con la complejidad de los problemas existentes en la estructura de la propiedad de la tierra, la vocación y el uso del área agrícola, así como los millones de personas con un arraigo a la tierra y que habitan el campo, fuente de vida y de bienestar.

En esa dirección, Zabala (2016) anota:

Desde comienzos del siglo, la FAO - Organización de las Naciones Unidas para la Agricultura y la Alimentación (2003) venía llamando la atención acerca de la necesidad de identificar una nueva concepción del desarrollo rural -coincidentes en Europa y América-, enunciando algunos lineamientos generales a tomar en cuenta por los gobiernos; así:

a) Acento en la dimensión territorial en contraste con la sectorial agraria, así como mayor conciencia de las distintas funciones y servicios prestados por la agricultura más allá del aspecto productivo;

b) Reconocimiento de los múltiples vínculos entre las pequeñas ciudades y el campo circundante y de la relación entre desarrollo urbano y rural;

c) Reconocimiento dela complementariedad entreagricultura y otras ocupaciones en la generación de ingresos rurales, de la generalización de la agricultura a tiempo parcial y del origen multisectorial del ingreso de muchas familias rurales;

d) Conciencia de la función residencial de las zonas rurales, en oposición a una percepción puramente productiva o recreativa de las mismas, que lleva a prestar atención a la provisión de servicios sociales y residenciales a las poblaciones rurales;

e) Reconocimiento de la creciente integración de las zonas rurales en los mercados (de productos, insumos, tecnología y mano de obra) y su incorporación al proceso de globalización, así como conciencia de la importancia de la competitividad territorial frente a la sectorial;

f) Atención al potencial económico que ofrecen los activos ligados al territorio, de tipo geográfico, histórico, cultural, paisajístico y ecológico; y 
g) Acento en la participación de los diversos agentes involucrados en el diseño y la aplicación de políticas y programas de desarrollo rural (p. 1). (Zabala, 2016, p. 12)

Bajo esa perspectiva, la economía campesina y la agricultura familiar se tornan en componentes de una problemática más compleja, que involucra elementos insospechados:

Así pues, el papel del medio rural dentro del conjunto de procesos del desarrollo de la economía, deberá darse en relación con otras funciones, muy distintas a las promovidas estrechamente desde comienzos del siglo XX. Estas funciones están relacionadas con temas como la conservación del medio ambiente, el equilibrio territorial, la utilización de tecnologías apropiadas, usos del suelo con funciones diferentes a la producción de materias primas (por ejemplo, para el eco-turismo, la recreación y la habitación) y la producción de alimentos limpios y orgánicos. (Zabala, 2016, p. 13)

Para Machado, citado por Zabala (2016, p. 13), esta es una visión holística del fenómeno del desarrollo rural, que supone

Vincular lo macro con lo micro, lo productivo con lo social, lo ambiental y lo político e introduce la conceptualización de género y el concepto de sostenibilidad y equidad (...) [así mismo, ello significa] una interpretación de la sociedad rural y sus procesos de articulación con el resto de la sociedad y el mercado mundial, una comprensión del mundo campesino en su perspectiva productiva, política, social y cultural. (Machado, 1995, p. 17)

Así las cosas, Machado (1995) define el desarrollo rural en los siguientes términos:

El desarrollo rural se concibe como un proceso dinámico de cambio acumulativo y de transformación de las sociedades rurales locales, que con la participación de los distintos actores sociales, permite diversificar tanto las actividades productivas y de generación de ingresos, como las formas de organización social y de participación política, y alcanzar desarrollos tecnológicos en medio de la diversidad cultural. (1995, p. 18)

\section{La asociatividad rural}

Ahora bien, los problemas de desarrollo rural son múltiples y complejos, por lo que no solo basta para resolverlos, con políticas públicas, orientadas a promoverlo, sino que también implica una activa participación de los protagonistas de dicho desarrollo, los campesinos o agricultores, que aran la tierra, desde diferentes perspectivas vitales.

Uno de los componentes del capital social es la confianza interpersonal, sobre la cual se construye la asociación entre sujetos autónomos con un proyecto compartido, conjugando la acción individual con la acción colectiva. En palabras de Zabala (2007):

La asociación es una ley natural y que entre los seres humanos adquiere una especial significación no sólo para la permanencia de la especie sino también para su propia convivencia y sustento, avanzándose hacia el perfeccionamiento de la conciencia colectiva. Con la asociación, al interior de la cual se produce el trabajo en cooperación, se generan nuevas realidades, transformaciones del entorno, mentalidades colectivas y evoluciona el pensamiento social. (2007, pp. 32-33) 
Tratándose de las posibilidades de fortalecer el capital social en una actividad tan heterogénea como las labores del campo y en una estrategia tan diversa como es el desarrollo rural,

La asociatividad agraria, (...), fue pionera en la formación del cooperativismo en el continente americano; muchos de los movimientos cooperativos (por ejemplo, en Brasil y Estados Unidos) se forjaron a través de la cooperación agraria. [De igual forma] en Bolivia, las cooperativas agrícolas fueron el motor de la transformación económica del país, aunque está muy limitada su actividad a la comercialización y prestación de servicios. En Ecuador existe una tradición de cooperativismo que ha sido acrecentado en la última década, siendo incentivadas las cooperativas dedicadas a la producción y comercialización de productos de tradición en el territorio (arroz, banano y café). (Zabala, 2007, p. 41)

Así mismo, el autor (2007) destaca la experiencia estadounidense, afirmando: "hay un inmenso número de cooperativas interviniendo en el mundo rural, sobre todo actuando como cooperativas de crédito agrícola, cooperativas de suministros, cooperativas de servicios, cooperativas de comercialización, asociaciones agrarias para la transformación de productos primarios y cooperativas de seguros".

De esa forma, la asociatividad se convierte en fuente y palanca del desarrollo rural, teniendo como actores principales tanto los productores que malviven en la economía campesina, como los agricultores familiares, que buscan superar las restricciones que el sistema capitalista les impone.

\section{ANTECEDENTES}

\section{El conflicto armado}

El conflicto armado en Colombia se inició aproximadamente hace 70 años con el asesinato de Jorge Eliécer Gaitán, candidato a la Presidencia; su muerte desencadenó un periodo que marcó notoriamente la historia del país, ya que este enfrentamiento entre el partido liberal y el partido conservador provocó más de 200000 muertes, desde el famoso "Bogotazo" (Arias, Camacho, Ibáñez, Mejía \& Rodríguez, 2014).

La estrategia para amortiguar este conflicto fue la creación del Frente Nacional que, aunque fue importante para disminuir la intensidad del conflicto entre los partidos, no incluía a otros movimientos políticos distintos a los llamados tradicionales; unido a esto, factores como la desigualdad del ingreso, la debilidad institucional y las disputas por las tierras, contribuyeron a la creación de los diferentes grupos guerrilleros (Arias, Camacho, Ibáñez, Mejía \& Rodríguez, 2014).

Debido a que estos grupos guerrilleros debían financiarse, el país se ha visto envuelto en situaciones de extorsión, secuestro, pago de vacunas, minería ilegal, narcotráfico, que han generado altos niveles de pobreza, violaciones, masacres, reclutamiento de menores, bajos índices de educación, entre muchas otras consecuencias negativas, especialmente en las zonas rurales donde se concentraron los grupos armados.

En Boyacá, desde el año 1978 se evidenció la presencia de guerrilla, siendo su geografía un factor relevante, ya que los grupos armados se concentraron en las partes altas 
del departamento. Los grupos paramilitares se crearon como defensa a los secuestros, extorsiones, homicidios, entre muchas otras atrocidades que estaba cometiendo la guerrilla en contra de los campesinos de la región, además de que algunos narcotraficantes se unieron a dichos grupos (El Espectador, 2013).

\section{La sustitución de cultivos}

Colombia se ha caracterizado por ser uno de los países que produce la mayor cantidad de coca que se consume en el mundo. Según el monitoreo de territorios afectados por cultivos ilícitos, en el país existían 144807 hectáreas de coca sembradas en el 2001, área que disminuyó en los siguientes años hasta el 2012, cuando se alcanzó el nivel más bajo de cultivos, que correspondió a 49000 hectáreas sembradas; sin embargo, en el año 2016 se evidencia un notorio aumento de cultivos -correspondiente a 146000 hectáreas de coca sembradas- como se puede ver en la gráfica 1.

Gráfica 1. Área de coca a 31 de diciembre 2001-2016

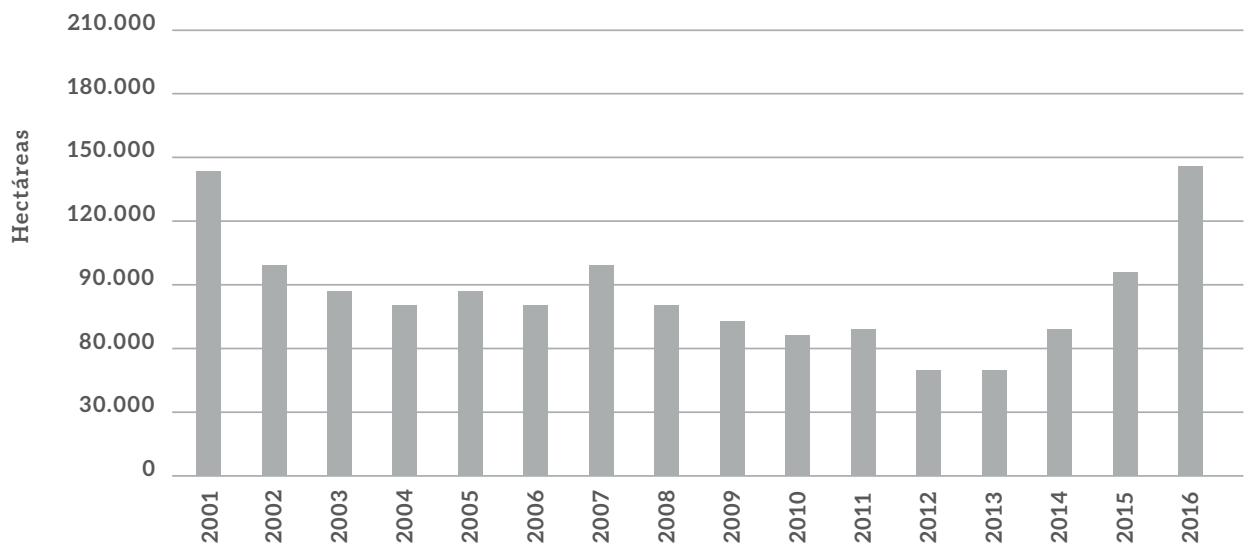

Fuente: Monitoreo de territorios afectados por cultivos ilícitos 2016.

Según el censo de cultivos ilícitos hecho en el 2016, Boyacá se encuentra entre los departamentos con menos de 50 hectáreas de coca sembradas. Esto se ha logrado gracias a la sustitución de cultivos que se ha venido presentando desde la puesta en marcha del Plan Colombia. En el occidente del departamento, los cultivos de coca se han sustituido principalmente por cultivos de cacao, aunque también de café, cítricos, guayaba, entre otros.

\section{El Plan Colombia}

El Plan Colombia (1999-2005) -creado con el propósito de combatir las drogas ilícitas, el crimen organizado y la violencia, así como también de reactivar económica y socialmente y fortalecer a los territorios afectados- se firmó en 1999 entre el Gobierno de Colombia y Estados Unidos, con una financiación de 10732 millones de dólares. Mediante este plan se busca mejorar las condiciones socioeconómicas de la población vulnerable, brindándole 
opciones diferentes de cultivos legales para aquellos que se han dedicado a la siembra de cultivos ilegales.

El acuerdo está conformado por cuatro elementos, que son la lucha contra el problema mundial de las drogas y el crimen organizado, la reactivación económica y social, el fortalecimiento institucional y la desmovilización, desarme y reintegración. Sus características son:

1. Lucha contra el problema mundial de las drogas: este componente tiene como objetivo la reducción de los cultivos ilícitos en el territorio nacional, el fortalecimiento de la capacidad de la fuerza pública, la modernización del sector de seguridad y defensa y el entrenamiento de la fuerza pública para garantizar los derechos humanos. Gracias a esto, las hectáreas de cultivos ilícitos en el país se redujeron aproximadamente en un 53 \% del año 2000 hasta el año 2005, mediante erradicación manual y aspersión de los cultivos; así mismo, los indicadores de violencia han disminuido notoriamente, como se muestra en la siguiente tabla:

Tabla 2.

Indicadores de violencia en Colombia 1990-2005

\begin{tabular}{|c|c|c|c|c|c|c|c|}
\hline INDICADOR & 1999 & 2000 & 2001 & 2002 & 2003 & 2004 & 2005 \\
\hline $\begin{array}{l}\text { Tasa de homicidios por } \\
100000 \text { habitantes }\end{array}$ & 58,6 & 62,7 & 64,7 & 65,9 & 53,2 & 44,0 & 39,1 \\
\hline Secuestros extorsivos & 2.587 & 2.089 & 1.208 & 1.709 & 1.257 & 758 & 378 \\
\hline Número de masacres & 168 & 236 & 236 & 115 & 94 & 46 & 48 \\
\hline Personas desplazadas & 31.635 & 331.982 & 331.982 & 424.927 & 221.413 & 162.936 & 169.111 \\
\hline $\begin{array}{l}\text { Miembros GAML } \\
\text { capturados }\end{array}$ & n.r. & n.r. & n.r. & 5.119 & 10.133 & 11.102 & 8.093 \\
\hline Bajas miembros GAML & n.r. & n.r. & n.r. & 1.877 & 2.265 & 2.520 & 2.192 \\
\hline $\begin{array}{l}\text { Ataques contra } \\
\text { infraestructura }\end{array}$ & n.r. & n.r. & n.r. & 979 & 676 & 358 & 449 \\
\hline
\end{tabular}

Fuente: Balance Plan Colombia 1999-2005 n.r.: No registra

2. Reactivación económica y social: en este componente se pretende promover las condiciones para la generación de empleo y la estabilidad social; ampliar las preferencias arancelarias, como elemento compensatorio de los efectos negativos del narcotráfico; favorecer la búsqueda de un acuerdo de libre comercio que amplíe las posibilidades de empleo; promover alternativas lícitas de desarrollo productivo regional y diseñar y poner en marcha la Red de Apoyo Social (RAS) ${ }^{1}$. Gracias a la seguridad con la que cuenta actualmente el país, se ha evidenciado un crecimiento en el PIB, disminución en la tasa de desempleo y en la inflación, como se puede evidenciar en las gráficas 2 y 3.

\footnotetext{
1. La Red de Apoyo Social está formulada con base en tres programas que son: 1) manos a la obra que proporciona ocupación transitoria a la población pobre, 2) subsidios condicionados a la población más pobre y 3) capacitación para jóvenes desempleados.
} 
Gráfica 2. Evolución del PIB (miles de millones de pesos) 2000-2017

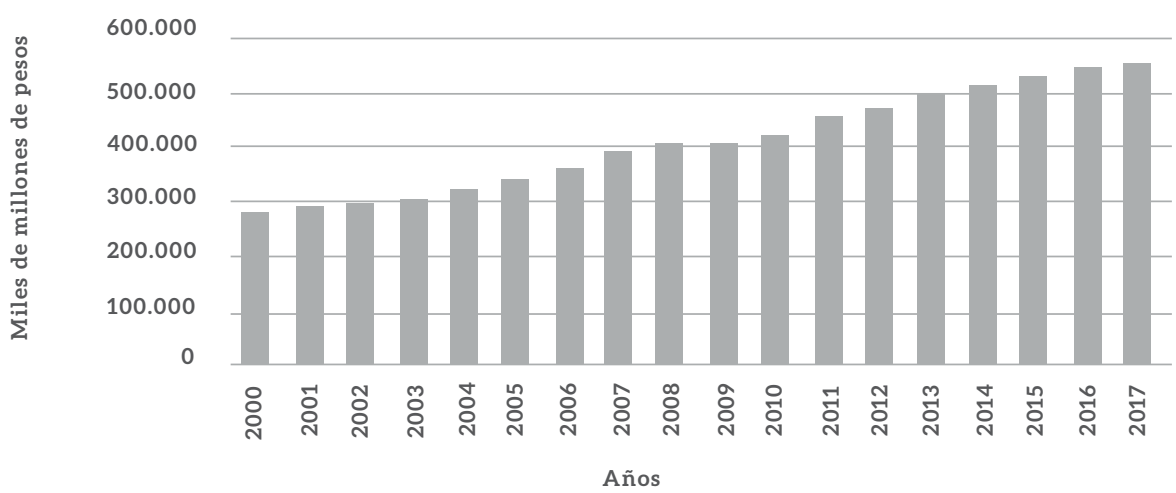

Fuente: Elaboración propia a partir de información del Banco de la República

Gráfica 3. Evolución de la tasa de desempleo, 2001-2017

\begin{abstract}
$20,0 \%$
$15,0 \%$

$10,0 \%$

$5,0 \%$

$0,0 \%$

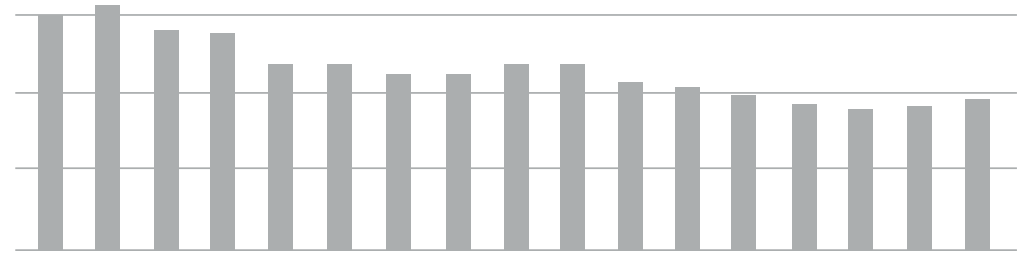

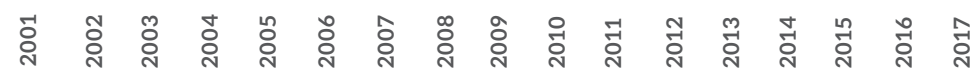

Años

Fuente: Elaboración propia a partir de información del Banco de la República

3. Fortalecimiento institucional: mediante este se busca proteger y recuperar el capital humano, físico, natural y social afectado por la violencia y el narcotráfico (inversión focalizada en regiones y poblaciones vulnerables), fortalecer y modernizar el servicio de justicia y reducir la impunidad y fortalecer la capacidad del Estado y la Fuerza Pública para proteger y garantizar los derechos humanos y el respeto al derecho internacional humanitario.

4. Desmovilización, desarme y reintegración: se pretende apoyar el proceso de desmovilización y reincorporación a la vida civil de los grupos armados al margen de la ley (GAML) así como también buscar la activa participación de la comunidad internacional en todas las etapas del proceso.
\end{abstract}




\section{MÉTODO}

Esta es una investigación exploratoria cualitativa, pues busca analizar el problema mediante la interpretación y comprensión de los procesos y resultados de la sustitución de cultivos ilícitos por el cacao.

En esta investigación se estudió la realidad en su contexto natural, intentando interpretar los fenómenos de acuerdo con las historias de vida de las personas implicadas; para lograrlo, utilizamos y recogimos material como entrevistas, encuestas, historias de vida, textos históricos que describen la rutina de las situaciones y problemáticas en la vida de los habitantes de Pauna.

La situación objeto de estudio en esta investigación fue el proceso de sustitución de cultivos de coca por el cacao en el municipio de Pauna y, por tanto, las consecuencias y cambios que trajo consigo en los diferentes sectores: económico, cultural y social, influenciados por los diferentes acontecimientos que vivió el municipio de Pauna.

El universo de estudio fueron 220 cultivadores afiliados a Aprocampa, 90 de los cuales participaron en el proceso de erradicación de la hoja de coca. Asimismo, se encuestaron 30 cacaoteros, participantes en el proceso de sustitución de cultivos, bien sea de manera directa o indirecta.

El instrumento de recolección de la información incluye variables nominales (sexo, ocupación), variables ordinales (grado), variables con escala (edad) para medir rangos y preguntas abiertas que permitan conocer más a fondo la situación social de los agricultores cacaoteros del municipio objeto de estudio.

\section{RESULTADOS}

Se presentan los resultados de las preguntas formuladas a los campesinos del municipio de Pauna, los cuales fueron analizados teniendo en cuenta aspectos generales (rango de edades, género, ocupación, nivel educativo) aspectos familiares (estructura familiar, cultivos actuales, área del predio, actividades desarrolladas en la parcela) y aspectos poblacionales (ingresos mensuales, servicios públicos, capacitaciones, entre otros).

\section{Generalidades de la población objeto de estudio:}

\section{Gráfico 2: Rango de edades}

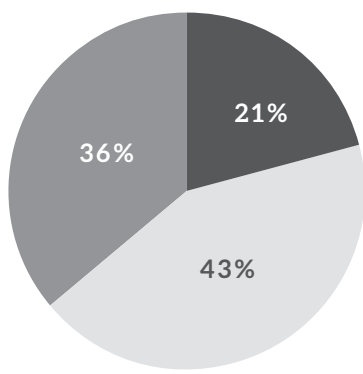

Entre 29 y 38 Entre 39 y 58 en adelante

Fuente: Elaboración propia

El $43 \%$ de la población encuestada se encuentra en el rango de 39 y 58 años, seguido de las personas mayores de 59 años (36 \%). El 21 \% está representado por las personas con edades entre 29 y 38 años. 


\section{Gráfica 3: Género de los encuestados}

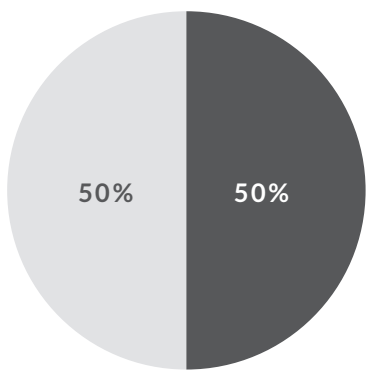

Fuente: Elaboración propia
Femenino

Masculino

Otro

Como se observa, la participación en la encuesta es de igual proporción (50\%), lo que permite obtener una información donde existe representatividad tanto de hombres, como de mujeres.

\section{Gráfica 4: Ocupación}

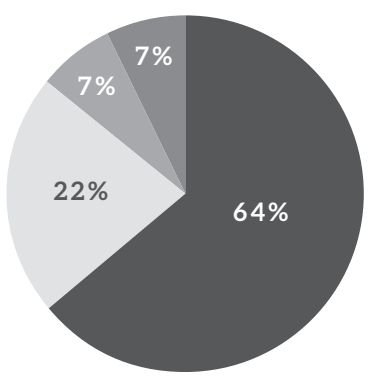

Fuente: Elaboración propia
Agricultor
Ama de casa
Técnico
No responde

Un 64\% de las personas encuestadas, se dedica a la agricultura, en tanto que un $22 \%$ de las mujeres se dedican a labores de ama de casa. De otra parte un 7\%, que poseen algún tipo de estudio técnico, se dedican a las actividades administrativas y auxiliares, y el restante $7 \%$ no dio respuesta a este punto de la encuesta, pero quizá se dedican a otro tipo de actividades, tales como minería, comercio formal e informal, entre otros.

\section{Gráfica 5: Nivel de estudios}

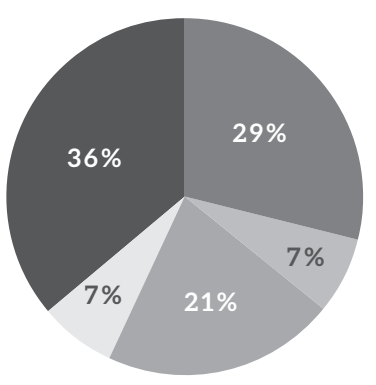

Fuente: Elaboración propia

\section{Básica Primaria Bachillerato \\ Básica Secundaria Técnico \\ No responde}

Se observa que un $64 \%$ de las personas encuestadas cursaron hasta básica primaria; el 22 \% logró culminar el bachillerato, en tanto que el $7 \%$ cursó hasta un nivel de básica secundaria sin superar el grado noveno, y el restante $7 \%$ concluyó nivel técnico aplicado. 
Gráfica 6: Conformación del grupo familiar

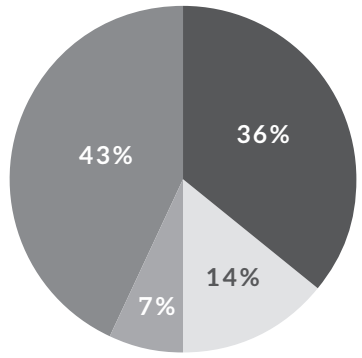
3 miembros
5 miembros
4 miembros
6 miembros

La mayoría de los grupos familiares está conformado por 6 personas (43\%), seguido por los núcleos familiares integrados por 3 personas (36\%), y la proporción restante la conforman entre 4 y 5 personas.

Fuente: Elaboración propia

Gráfica 7: Ingresos mensuales percibidos

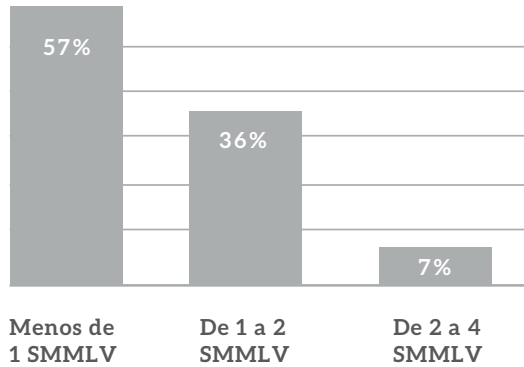

Fuente: Elaboración propia

El57\%deloscampesinosencuestados recibe ingresos mensuales menores a 1 SMMLV, y tan solo el $7 \%$ percibe más de 2 SMMLV.
Gráfica 8: Tamaño de la parcela

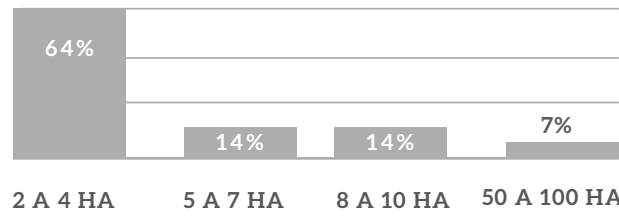

Fuente: Elaboración propia

Los predios donde se desarrollan las distintas actividades agropecuarias poseen un área pequeña. El $64 \%$ es propietario de parcelas cuyo tamaño fluctúa entre 2 a 4 hectáreas. Así mismo, el $14 \%$ posee predios entre 5 a 7 hectáreas; mientras que el $21 \%$ restante posee 8 hectáreas, hasta alcanzar 100 hectáreas.

Gráfica 9: Actividades desarrolladas por los campesinos

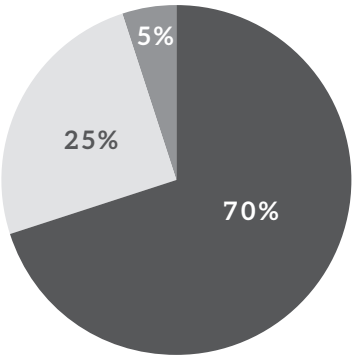

Fuente: Elaboración propia
Cultivos Ganadería Pecuaria

El $70 \%$ de los encuestados destina su parcela en la explotación agrícola, mientras que el $25 \%$ se usa en la explotación estrictamente ganadera. El restante $5 \%$ de los encuestados dedica su parcela a la explotación pecuaria (peces, cabras, cerdos, etc.). 
Gráfica 10: Cultivos en la actualidad en los predios de los campesinos encuestados

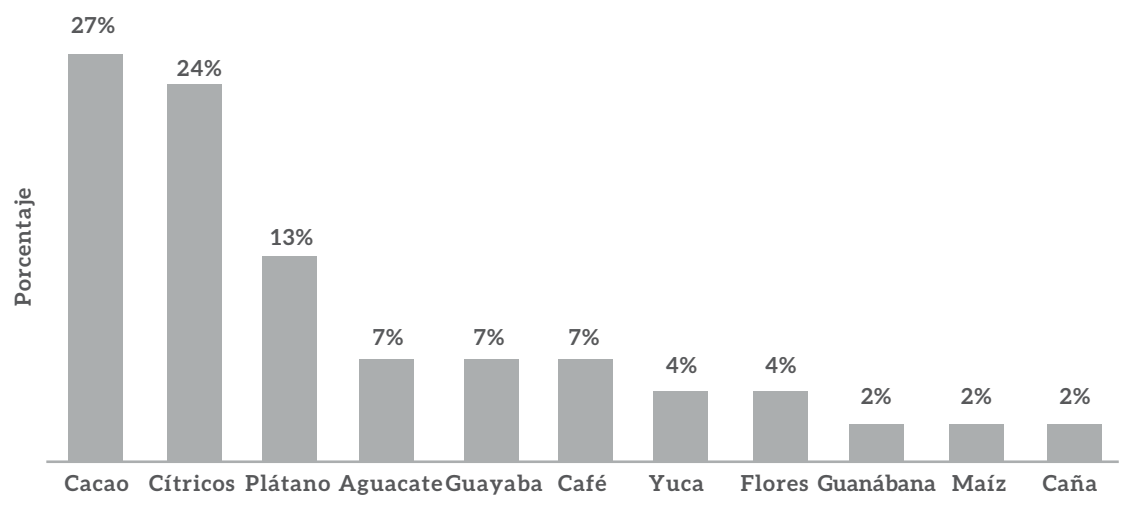

Fuente: Elaboración propia

La alta biodiversidad de los cultivos se revela al considerar que en el $27 \%$ de los predios está presente el cultivo de cacao; mientras que en el $24 \%$ se cultivan cítricos. En el resto de las parcelas (51\%) se siembra plátano, aguacate, guayaba, café, yuca, flores, guanábana, maíz y caña panelera.

\section{Gráfica 11: Servicios públicos disponibles}

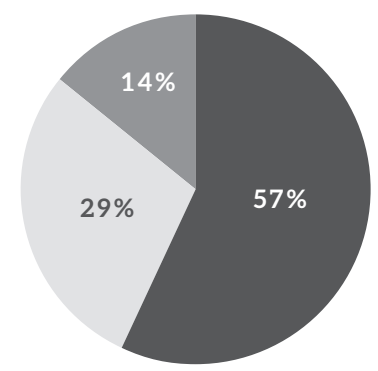

Fuente: Elaboración propia
Energía Agua potable elétrica y energía eléctrica

Agua potable, alcantarillado, energía eléctrica

El $57 \%$ de los encuestados cuenta solamente con servicio de energía eléctrica de manera permanente; en contraste, $29 \%$ dispone de servicios de agua potable y energía eléctrica. Los demás (14\%), cuentan con todos los servicios públicos domiciliarios (agua potable, alcantarillado y energía eléctrica).

\section{Gráfica 12: Afectación de las víctimas por la violencia}

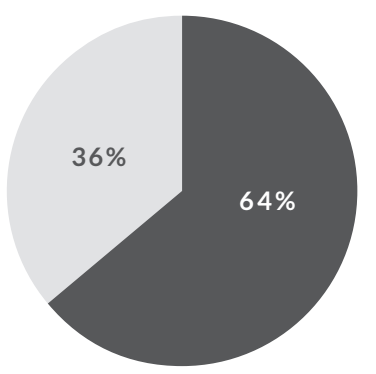

Sí

No

Fuente: Elaboración propia 


\section{Gráfica 13: Comercialización de productos}

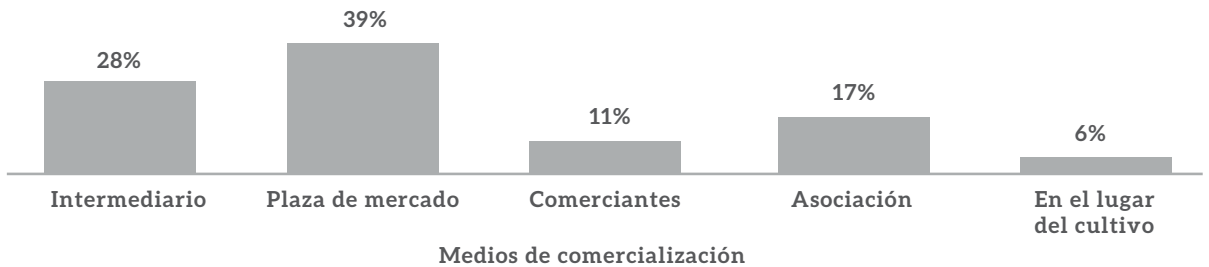

Fuente: Elaboración propia

El 50\% de los encuestados comercializa sus productos con intermediarios, el $28 \%$ lo hace cada domingo en la plaza de mercado. Cabe destacar, que el $17 \%$ comercializa sus productos por medio de la Asociación de Productores de Cacao del municipio de Pauna. Por último, el $6 \%$ vende sus productos en el lugar del cultivo.

Gráfica 14: Limitaciones para la comercialización de productos

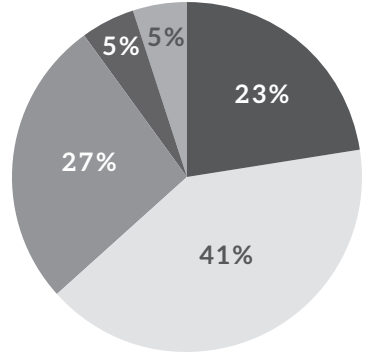

Fuente: Elaboración propia

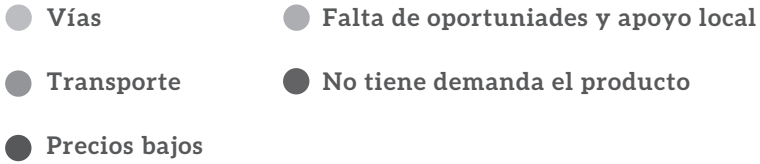

El $41 \%$ de los encuestados considera que las limitaciones de comercialización están relacionadas con la infraestructura vial, mientras que el $27 \%$ se las atribuye a la falta de transporte adecuado. Por otro lado, el $23 \%$ lo relaciona con los precios bajos de los productos. El restante $9 \%$ lo asocia con la baja demanda del producto comercializado, ocasionado por la falta de oportunidades y apoyo local.

\section{Gráfica 15: Conocimiento del plan Colombia}

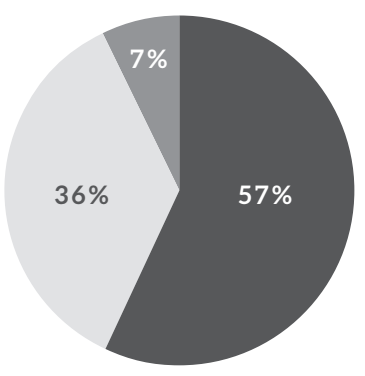

Fuente: Elaboración propia
No

No responde

El $57 \%$ de encuestados conoció y fue beneficiado por el Plan Colombia, mientras que el 36 \% no lo conoció. El $7 \%$ de los encuestados no sabe ni responde acerca de este tema en su vereda. 


\section{Gráfica 16: Cultivos existentes antes del Plan Colombia}

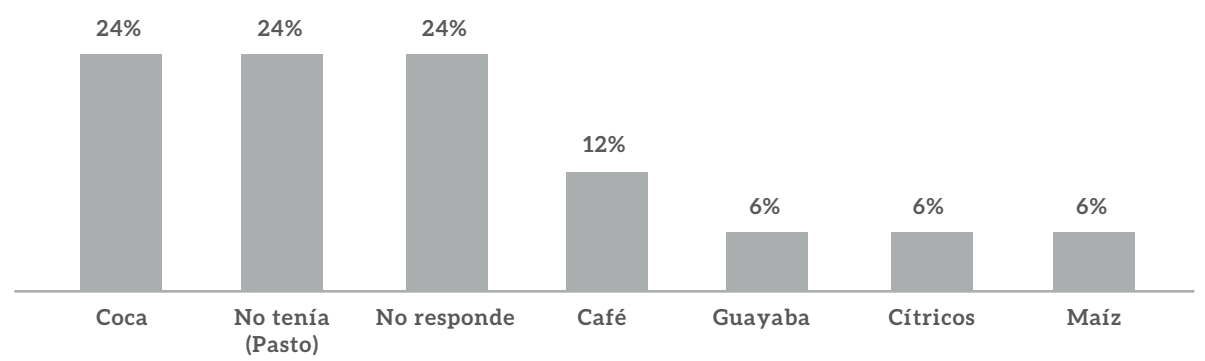

\section{Fuente: Autoras}

El 24 \% de los encuestados, declaró que había sembrado cultivos ilícitos, otro 24 \% a la siembra de pasto; en tanto, que un $12 \%$ afirmó cultivar café. Los restantes se dedicaban a cultivar guayaba, cítricos y maíz. Es revelador que el 24 \% de los encuestados prefirió no responder.

\section{Gráfica 17: Vinculación a asociaciones de agricultores}

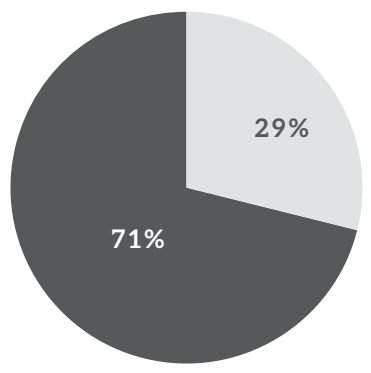

Sí No

Fuente: Autoras

El $71 \%$ de los encuestados está vinculado a alguna asociación de agricultores, entre las cuales se encuentra Asociación de Cacaoteros, de Cafeteros y de Frutas.

\section{Gráfica 18: Capacitaciones en temas agropecuarios}

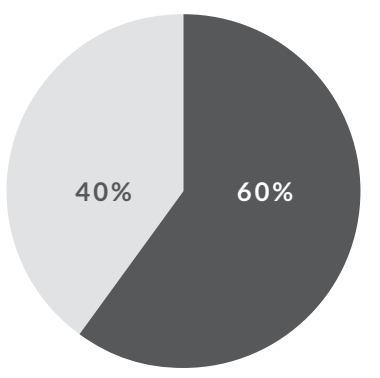

Sí No

El $60 \%$ de los encuestados manifiesta haber recibido capacitación por parte del Estado para realizar el proceso de sustitución de cultivos, y el 40 \% declaró que no han recibido ningún tipo de capacitación.

Fuente: Autoras 
Gráfica 19: Frecuencia de la capacitación

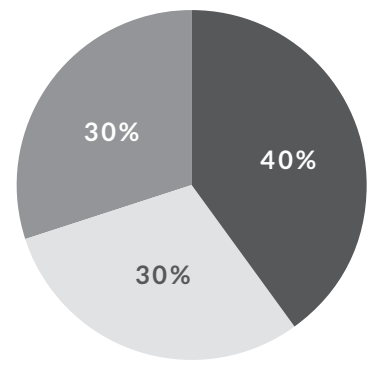

Mensual Semestral Anual

Fuente: Autoras

Gráfica 20: Beneficios de la erradicación de cultivos

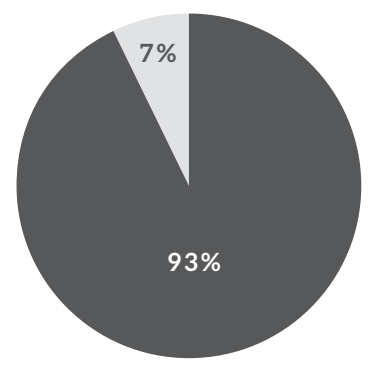

Fuente: Autoras
Sí No sabe

El impacto de los programas de apoyo a la sustitución de cultivos se refleja de manera notoria al considerar que desde el año 2003, el 93 \% de los encuestados respondió que han sido muchos los beneficios que le ha traído al municipio la erradicación de cultivos ilícitos. Solo el 7 $\%$ no sabe o no ha percibido los beneficios directos de la erradicación en sus veredas.

\section{DISCUSIÓN}

En el municipio de Pauna se evidencia que las actividades económicas se basan en un $64 \%$ en la agricultura. Además, como se observa en la gráfica 9, se confirma que el 70 $\%$ de los encuestados dedica sus labores a siembra de cultivos y solo el $30 \%$ se dedica a labores pecuarias (ganadería, piscicultura, porcicultura, etc.). Asimismo, que un $22 \%$ de las mujeres se dedica a las actividades del hogar y la agricultura. Por otro lado, el $7 \%$ de los encuestados ha recibido educación técnica y el 7 \% se dedica a diversas actividades formales e informales.

Los cultivos de uso ilícito fueron desarrollados especialmente por familias campesinas en predios de tamaño pequeño, como se refleja en la gráfica 8, que muestra que el $69 \%$ de los encuestados posee predios entre 2 y 4 hectáreas. Además, se evidencia una alta participación de personas entre 39 y 58, representando el $43 \%$ de los encuestados. El $36 \%$ de los encuestados tiene edades superiores a los 59 años, lo que demuestra que la mano de obra es cada vez más escasa, ocasionando cambios importantes dentro de la estructura familiar, debido a que los varones de las familias se trasladan a las cercanías de las minas, ausentándose por largas temporadas de sus hogares, dada la creciente oferta 
de actividades económicas alrededor de la extracción minera, mientras las mujeres continuaron en el rol doméstico agricultor.

Como se aprecia en la gráfica 4, el nivel educativo de los encuestados es muy precario, ya que solamente el $21 \%$ de los encuestados logró culminar sus estudios como bachiller. El 29 \% culminó la básica primaria, y solo el $7 \%$ tuvo la posibilidad de culminar estudios técnicos. Además, el 36 \% no tiene claro su nivel educativo y el 7 \% cursó algún grado de educación básica. Lo anterior refleja que la educación es un componente social determinante en el desarrollo del municipio, dado que tiene gran impacto la apropiación de saberes académicos, superando el conocimiento empírico y ancestral de estas comunidades. Estudios desarrollados en el municipio (Hernández \& Monroy, 2017) identificaron que el impacto se ha reflejado en el cambio de uso de la tierra, la ampliación de la frontera agrícola y el impacto medioambiental. Adicionalmente, en las comunidades campesinas ha sido evidente la transformación social e institucional (Hernández \% Monroy, 2017).

De acuerdo con estudio realizados en el municipio de Pauna por Hernández y Monroy, entre los años 2005 y 2015 la presencia gubernamental se institucionaliza, mediante el Programa de Desarrollo Alternativo 2003-2006 (Conpes 3218, PDA), que tuvo como estrategia eliminar las drogas ilícitas. Este programa se implementó a través de tres componentes: el primero, mediante proyectos productivos (agroforestales y agrícolas) que generaran ingresos para asegurar el alimento de las familias. El segundo estuvo centrado en las familias guardabosques que compromete a las familias campesinas a vincularse al programa y a conservar los ecosistemas naturales del país y el cuidado del agua. Por último, el fortalecimiento institucional, desarrollo social y monitoreo, con el cual se busca mejorar los vínculos con el Estado y coordinar entre instituciones el compromiso de cumplir con los objetivos del programa (Hernández \& Monroy, 2017).

En departamentos como Nariño y Putumayo participaron familias pertenecientes a los territorios colectivos, líderes y dirigentes comunitarios que discutieron alternativas para la erradicación de los cultivos de coca, y, al igual que en el municipio de Pauna, la reconstrucción de los valores étnicos y la integración de sus comunidades mediante un plan de desarrollo alternativo que permitió brindar insumos agrícolas, investigación y capacitación a agricultores como lo refieren Sánchez et al. (2006). En la gráfica 18 se evidencia que los procesos de acompañamiento son altamente eficaces, comoquiera que el 60 \% de los encuestados recibe capacitación técnica, mientras que solo el 40 \% no la ha recibido. Quizá esto esté asociado a la distancia entre veredas, la poca posibilidad de tiempo para asistir, además de las limitaciones en el transporte intraveredal. Estas capacitaciones son recibidas con una frecuencia semestral para el $40 \%$ de los encuestados, mensual para el 30 \% y una proporción idéntica, con una periodicidad anual. Esta situación puede estar relacionada con la poca participación de instituciones del Estado en el municipio y la falta de recursos por algunas entidades presentes en la zona como el SENA y las entidades prestadoras del servicio de asistencia técnica agropecuaria del municipio (Epsagro).

Estudios realizados por Hernández y Monroy establecieron que el programa familia guardabosques proporcionaba dinero que era utilizado para estimular el desarrollo del programa en cultivos como café, cacao, forestales, silvopastoriles (ganadería) y caña. En la gráfica 10 se muestra que las fincas poseen alta biodiversidad, siendo el cacao el cultivo que tuvo mayor acogida, por las condiciones del clima de la provincia; estas fueron siembras nuevas, es decir, cuando se hace enjertación con malaya y cambio de copas (Hernández \& Monroy, 2017), lo cual coincide con los resultados de nuestra investigación, pues así lo manifestó el 27 \% de los encuestados que posee cultivos de cacao. 
De acuerdo con Hernández y Monroy (2017), este proceso permitió legalizar las actividades de los campesinos y promovió el bienestar de los habitantes de esta provincia; por esta razón, es de suma importancia resaltar su papel no solo para la erradicación de los cultivos ilícitos, sino como articulador de procesos sociales como la conformación de cooperativas de productores, como el caso de la Asociación de Productores de Cacao del municipio de Pauna (Aprocampa), en que el $71 \%$ de los encuestados afirma hacer parte de asociaciones, microempresas, formación de líderes, integración a la economía legal, como es el caso del convenio con Casa Luker para la compra del cacao (Hernández \& Monroy, 2017).

Estudios realizados en Nariño y Putumayo (Sánchez et al., 2006), relacionados con la sustitución de coca por cacao, identificaron que la falta de políticas se refleja en ausencia de investigación, de asistencia técnica, de transferencia de tecnología, entre otros factores. Además, en la deficiencia del sistema de créditos agropecuarios, bajos precios de los productos de cacao, la ausencia de acompañamiento de socios empresariales y la falta de mercado para otros productos tradicionales. En la gráfica 13 se observa que, para el caso de Pauna, dentro de los distintos canales de comercialización de los productos, la mayoría (50 \%) manifestó que se concentra en la plaza de mercado, seguido de un 28 $\%$, que vende sus productos en la plaza de mercado muy rara vez; además, el $17 \%$ los comercializa en la Asociación. Asimismo, el 6 \% respondió que sus productos quedan en manos de los intermediarios, que van a cada una de las veredas del municipio a comprar los productos al precio que a ellos mejor les beneficie, sin pensar en el impacto económico que generan en el agricultor campesino. Lo anterior se refleja en la gráfica 7, que revela que los ingresos mensuales son menores a 1SMMLV y, en muchas ocasiones, como se evidencia en la gráfica 6, no alcanza para la manutención de las familias compuestas por 3 o más personas.

Al igual que en el municipio de Pauna, en muchas regiones del país, los principales problemas del sector agropecuario tradicional, según los consejos comunitarios, están representados en la baja productividad de los cultivos tradicionales, debido al deficiente nivel tecnológico, la fuerte presencia de plagas y enfermedades, especialmente en el cultivo del cacao, y la falta de empresas comunitarias para comercializar los productos, lo que se refleja en ingresos muy bajos para las familias campesinas (Sánchez et al., 2006). Por esta razón, ha sido muy importante el papel de los cultivos de pancoger, como lo muestra la gráfica 10, en la que la biodiversidad alimenticia se sustenta en cultivos como cítricos (24\%), plátano (13\%), aguacate, guayaba y café (7 \%) y cultivos como guanábana, maíz y caña (2\%).

Como se aprecia en la gráfica 11, la calidad de servicios públicos es muy precaria, teniendo en cuenta que el municipio de Pauna tiene 28 veredas, y solo el $57 \%$ de los encuestados cuenta con energía eléctrica de manera permanente. A su vez, el $29 \%$ de la población cuenta con servicio de agua potable y energía eléctrica. Únicamente el 14 \% dispone de energía eléctrica, agua potable y alcantarillado, aunque de mala calidad, debido a que algunas veces las lluvias provocan que el agua sea turbia. A ello se agrega falta de adecuación y puesta en funcionamiento de sistemas de saneamiento de agua en la planta de tratamiento, la cual está abandonada hace más de 5 años. Los consejos comunitarios concluyeron que en los territorios colectivos el suministro de agua potable y los sistemas de saneamiento básico son limitados e insuficientes o no existen, además muchos municipios no tienen siquiera acueducto veredal. A lo anterior se suman las limitaciones para la comercialización, como se observa en la gráfica 14: el 41 \% de los encuestados afirmó que las limitaciones se relacionan con fallas en la infraestructura vial, el $27 \%$ por falta de transporte, y el $23 \%$ por precios bajos, impuestos muchas veces 
por intermediarios. El panorama en Pauna es positivo en comparación con los municipios de Nariño y Putumayo, donde el 100 \% de la población que hace parte de los consejos comunitarios no toma agua potable ni cuenta con servicio de alcantarillado ni energía eléctrica (Sánchez et al., 2006).

De igual forma, los resultados coinciden con estudios precedentes, teniendo en cuenta que el municipio de Pauna es un territorio de topografía variada, altamente montañosa, que además posee una precariedad en la infraestructura vial, que da acceso a las 28 veredas del municipio. Aquí se presentan similitudes con los territorios colectivos de los consejos comunitarios de Nariño y Putumayo, en los que la infraestructura social existente está en mal estado y es deficiente, las vías de penetración son escasas y no hay servicio de energía eléctrica permanente. En este aspecto de suministro de energía eléctrica, el $57 \%$ de los pobladores encuestados cuenta con este servicio, pero, en épocas de lluvias se ve interrumpido en muchas de las veredas alejadas del casco urbano.

El cacao es muy importante para el proceso histórico de posconflicto en que está comprometida la sociedad colombiana en este momento, ya que los cultivos de cacao, debido a su posición geográfica se encuentran en las zonas que fueron más golpeadas por el conflicto armado por más de medio siglo, y donde se localiza la mayor concentración de producción de cultivos ilícitos del país. Es por esto, que en Pauna han sido tan bien acogidos los programas de sustitución, ya que fomentan la agricultura familiar, que incorpora la mano de obra familiar, fuente de biodiversidad en los territorios, generando así un alto impacto medioambiental y social con la mitigación de la violencia, al ser cultivos lícitos que incluyen a toda la población en la legalidad. Estos beneficios se reflejan en la gráfica 12, que revela que el $64 \%$ de la población no fue víctima directa, en tanto que el $36 \%$ fue víctima directa.

\section{CONCLUSIONES}

El perfil de los encuestados es: 43 \% de los agricultores tiene edades entre 39-58 años, el nivel de escolaridad es básica primaria; el núcleo familiar del $43 \%$ está integrado por 6 personas, y los ingresos obtenidos no sobrepasaban un salario mínimo mensual.

Las características sobresalientes de los predios de propiedad de los encuestados son: el 64 \% tiene parcelas cuyo tamaño es de 2-4 hectáreas, el 70 \% lo destina a la explotación agrícola, y el 27 \% declaró tener cultivos de cacao. Todo lo anterior muestra que la estructura de estos predios rurales se inscribe dentro de la economía campesina en transición a la agricultura familiar, en la que los hogares enfrentan problemas de acceso a los servicios públicos domiciliarios. Solo el $14 \%$ tiene cobertura de agua potable, alcantarillado y energía eléctrica.

A pesar de que el municipio de Pauna es uno de los territorios azotados por la violencia, resulta paradójico que el $64 \%$ de los encuestados manifestó no haber sido víctima de la violencia. Por otro lado, una de las limitaciones más severas de los cacaoteros de Pauna es la comercialización, comoquiera que el 50 \% de los campesinos comercializa sus productos a través de intermediarios, vendiendo solo el 17 \% por intermedio de la asociación de productores, como es el caso del cacao. La infraestructura vial representa el 41 \% de las limitantes, según la percepción de los agricultores encuestados, seguida por las dificultades de transporte (27\%). 
Es muy diciente que solo el $24 \%$ de los encuestados reconoció haber sembrado cultivos ilícitos antes de la implantación del Plan Colombia. Sin embargo, el 57 \% expresó conocer y haberse beneficiado del mismo. Es sintomático de los cambios sociales en Pauna, que en la percepción del $93 \%$ de los encuestados se refleje el reconocimiento de que el programa de erradicación de cultivos ilícitos fue muy benéfico.

La erradicación de cultivos ilícitos y su sustitución por cultivos como el cacao ha traído indudables beneficios, al ofrecer alternativas de legalidad y fuentes de ingresos a la economía familiar, y contribuir al desarrollo rural y a la cohesión social, a lo que se añade un factor alentador para este tipo de economía campesina: el $71 \%$ de los encuestados pertenece a una asociación de agricultores.

Pese a que el $60 \%$ contestó que ha accedido a programas de capacitación, se requiere un continuo apoyo, participación y acompañamiento de entidades como el Sena, Epsagro y otras instituciones del Estado.

La Asociación de Productores de Cacao del Municipio de Pauna (Aprocampa), con distintos reconocimientos nacionales e internacionales, ha sido determinante en las transformaciones ocurridas en el municipio, además de ser una asociación pionera en la transformación del cacao con la apertura de tiendas comerciales especializadas. La consolidación de estas iniciativas no podrá darse si no van acompañadas de permanentes políticas gubernamentales de estímulo al desarrollo rural, que involucran factores económicos, políticos, sociales, ambientales, institucionales, entre otros.

La economía campesina es clave en el desarrollo rural. La asociatividad es fundamental en el bienestar de los pobladores del campo, por lo que es muy importante que los campesinos sean reconocidos y visibilizados, convirtiéndose en beneficiarios de las políticas públicas con enfoque territorial, para así desterrar la violencia y la desigualdad.

El presente estudio de carácter exploratorio podrá ser el punto de partida para adelantar otras investigaciones que se centren en componentes específicos de la estrategia de desarrollo cacaotero y la superación de los problemas heredados de los cultivos ilícitos.

\section{REFERENCIAS BIBLIOGRÁFICAS}

Arias, M., Camacho, A., Ibáñez, A. M., Mejía, D. \& Rodríguez, C. (2014). Costos económicos y sociales del Conflicto en Colombia ¿Cómo construir un posconflicto sostenible? Bogotá: Ediciones Uniandes.

Bartra, A. (1982). El comportamiento económico ce la producción campesina. México: Universidad Autónoma de Chapingo.

Chayanov, A. (1974). La organización de la unidad económica campesina. Buenos Aires, Argentina: Nueva Edición.

Departamento Nacional de Planeación, Departamento de Justicia y Seguridad. (s.f.).

Plan Colombia. Obtenido de https://colaboracion.dnp.gov.co/cdt/justicia\%20seguridad\%20y\%20 gobierno/bal_plan_col_espan ol_final.pdf 
Diez, M. (2013). Campesinado: definiciones analíticas y contextos históricos. Estudios Rurales (4), 153-167.

El Espectador. (27 de julio de 2013). Así fue la génesis del paramilitarismo. Obtenido de https://www. elespectador.com/noticias/judicial/asi-fue-genesis-del-paramilitarismo-articulo- 436386

Esteve, M. (2009). Tierra y agua para poder producir y vivir: el movimiento campesino cordobés. Theomai Journal, 20, 1-15.

Gonzalves, G. (2007). Economía campesina y economía comunitaria: apuntes para analizar las experiencias en proyectos de desarrollo rural. En Documento del Seminario Comunidad y Economía del Desarrollo (p. 13). Santa Cruz.

Llambi, L. (1988). Small modern farmers: Neither peasants nor fully-fledged capitalists? The Journal of Peasant Studies, 15(3), 350-372.

Machado, A. (1995). Reestructuración y desarrollo institucional en el cooperativismo agropecuario. Tendencias de desarrollo. Bogotá, Colombia: Fundecoop.

Martínez, J. \& Acevedo, A. (2016). Una mirada al mundo de la agricultura y el desarrollo rural. En La agricultura familiar en Colombia. Estudios de caso desde la multifuncionaldad y su aporte a la paz (págs. 9-29). Bogotá: Ediciones Universidad Cooperativa de Colombia.

Oficina de Naciones Unidas contra la Droga y el Delito. (Julio de 2017). Monitoreo de territorios afectados por cultivos ilícitos, 2016. Oficina de Naciones Unidas contra la Droga y el Delito.

Pimbert, M. (1995). La necesidad de otro paradigma de investigación. Biodiversidad, 2(2), 20-26.

Sevilla, E. \& González, M. (2004). Sobre la evolución del concepto de campesinado en el pensamiento socialista: una aportación para la vía campesina. Brasilia.

Valderrama, M. \& Mondragón, H. (1998). Desarrollo y equidad con campesinos (Vol. 2). Bogotá: Tercer Mundo.

Yoder, M. (1994). Critical chronology and peasant production: small farm forestry in Hojancha. Guanacaste, Costa Rica.

Zabala, H. (2007). Asociatividad, redes sociales y desarrollo territorial. Unircoop, 5(1), 28-50.

Zabala, H. (2016). Economía agraria y asociatividad coopertaiva en Colombia. En F. U. Amigó, Economía agraria y asociatividad cooperativa en Colombia. Medellín, Colombia. Obtenido de https://www.unodc.org/documents/colombia/2017/julio/CENSO_2017_WEB_baja.pdf 